\title{
The Utilisation of Physical Diagrams for the Modelling of Electric Vehicle Charging
}

\author{
Vladislav Sít’ar̆, Tomáš Vysloužil \\ Jan Evangelista Purkyne University in Usti nad Labem, Faculty of Mechanical Engineering, Institute of Machines and \\ Power Engineering, Pasteurova 3334/7, 40096 Usti nad Labem, Czech Republic. E-mail: vladislav.sitar@ujep.cz, to- \\ mas.vyslouzil@ujep.cz
}

\begin{abstract}
The paper describes the utilisation of several physical diagrams for the modelling of chosen electric car charging in alternative simulation software for an electric power system. The description and evaluation of possible ways for modelling of electric vehicle charging with the results that are sutable for basic energetic analysis are performed, and for all cases given models are used in chosen simulation circuits. Modelling of electric vehicle charging becomes important with the increasing trend of used electric cars and installed charging stations. Electric vehicle charging represents the rising amount of loads with nonstandard behaviour which has been connected to the distribution power grid. The behaviour of electric cars during charging is not the same as a standard passive load, which can be defined by consumed active power for a specified period of time. The charging process has a specific behaviour, which is derived by the type of a charging station and communication with the battery in the car. While charging the distribution grid is influenced. The undesirable occurred phenomena are unbalanced powers and generation of harmonics, which can cause voltage and current asymmetry in the grid and subsequently worsen electrical voltage quality. Furthermore, the load capacity of distribution lines is reduced and therefore the steep increase of loading limits their safety and economic usage.
\end{abstract}

Keywords: Electric vehicle charging, Load modelling, Constant impedance load, Constant current load, Constant power load

\section{Introduction}

Recently, methods for the estimation of the impact of a large number of electric vehicle (EV) to the electric power system have been used. For example, using the Monte Carlo method for describing the interaction of EV Chargers is known $[1,2]$. Next, current ways are focused on the modelling of EV chargers. The behaviour of a charging station while charging has been usually assessed of power electronic devices branch and their control.

The charging of EV is realized in several ways depending on the form of power and speed of charging. Nowadays, three speeds of wired charging are distinguished - slow, fast and rapid. The higher power is transferred, the shorter time for fully charging is needed. Form of the supplied power depends on the connector type of a particular EV, i.e. where is located the output rectifier whether in a car or in a charging station. Generally, the current flows from AC network, although the directly available DC voltage from microgrids expands more and more [3]. The AC voltage is rectified in a rectifier into the $\mathrm{DC}$ one, after that it is transformed in an isolated DC/DC converter with output rectifier at medium frequency into the DC voltage of an appropriate level for the charging. The AC charging stations are single or three-phase and they are designed for all three speeds of charging, i.e. in different power ratings, however the rapid AC charging stations occur rarely. The rapid charging is provided by the DC charging stations.

Using of power converters enables the controlling of the output voltage and current. The modelling of such system that takes account of switching processes of the semiconductor power devices has been widely used, especially for consumed current analysis, mainly for current magnitude and harmonic content [4], or for power flow analysis [5]. However, the demands on the hardware and software equipment, on the detailed knowledge of charging system parameters and on the way of control are relatively high and the simulation models are complex.

Modelling of electric vehicle (EV) charging by physical diagram as a general physical load is not currently in use. This specific type of load is not possible to model by ordinary impedance with appropriate parameters but a typical charging process has to be considered. It can be said, that with the current electromobility growth, the requirement for reliable modelling of EV charging will increase not only via a system of equations but also by physical diagrams.

Modelling by using physical diagrams can be useful for other simulation branches. Such modelling can be used for actual problems in a power system. For instance, to assess the ampacity and thermal loading of distributed cable lines, which feed the charging stations, described in more details in [6]. This problem is also bound to the ampacity of transmission lines, because the increase of distribution lines will lead to a higher transmission of power by transmission lines, further described in [7]. It is possible to use it in complex modelling methods and also for batteries in the thermal modelling of EV, more information described in [8]. Consecutively, its next utilisation is possible to aim at materials of electric cables and lines, other technical equipments and also for using convenient production technologies for them. In this case, the electrical conductivity and structure of materials influence mechanical and electrical properties of EV chargers internal devices [9]. Finally, the electrical conductivity of EV charger internal devices and supply cables affects the level of consumed currents. The structure of the materials limits their dynamic electrical and also mechanical loading. A steep current increase or intense deviations may 
cause dynamic stress of current paths. It may leads to the vibrations or oscillations current-conductive elements inside EV chargers [10, 11]. This problem may be also solved by physical diagrams, where dynamic changes of current excite mechanical forces.

The consumed power by the EV charger or a battery from the grid has many specifics. They are derived from the type of charging station and the system of charging. Furthermore, they are derived from the sophistication of the system and its control and their communication with the EV. When connecting the EV to the charging station according to the type of the station and the charging method, the unbalanced phases load may arise. It is true also for three phases charging. Simultaneously, the harmonics are generated to the grid and their content and magnitude depend on the charging time and also on the use of filtration in the charging station. In addition, current peaks may appear at the start of charging.

For the appropriate creation of a model it is necessary to consider all important influences which are not always possible. All influences do not have to be considered in certain cases. Therefore, it is suitable to create several model types which always respect requested behaviour attributes, and which can be modelled as simple as possible. Engaged modelling problems will focus only on the EV charging in a view of magnitude and the time electric power is consumed from the grid. The EV and charging station are considered as one simple load with defined load characteristic.

\section{Load modelling}

For the load modelling, in other words, the power consumption of the charging station, two possible ways have been tested using the physical diagram. The static models or dynamic models can be used. If we take the process time of power consumption into consideration, it is suitable to use the static models. A transient state at the beginning of charging and its dynamic are irrelevant for solving the problem. Such modelling may be imagined as a combination of RLC elements in parallel or serial connection. Thus, such a load model is represented by passive elements in each phase with a certain size and character of impedance.

Consumed active and reactive powers are usually the input values for static models, which correspond to the voltage magnitude and its frequency at the connection point. In accordance with the load behaviour when voltage changes, the load models are divided into three nominal types. They are models defined by constant impedance, constant consumed current or constant consumed powers [12-16]. Their combination or behaviour approximation arises from polynomic and exponential models, further detail is mentioned in [12-15]. Due to the definition of an exponential model with regard to a mathematical description of consumed current or power waveforms, the model will not be considered for testing.

The active power is the main part of the total power consumed while charging. Reactive power plays only a marginal part and its magnitude matters only in nonstandard conditions while charging. Therefore, all the mentioned theory below is only related to the active power.

\section{Constant impedance load}

Active power consumed changes with the power of voltage value in this model $P\left(V^{2}\right)$. It is sometimes called a model with constant admittance. Mathematical formula is introduced in eq. (1). If the voltage is increased by $\Delta V$, consumed active power also increases according to this voltage change with square and vice versa. The total impedance of load remains constant. If the voltage is not changed $V=V_{n}$, active power corresponds to its nominal value $P=P_{n}$.

$$
\frac{P}{P_{n}}=\left(\frac{V}{V_{n}}\right)^{2}
$$

Where:

$P_{n}[\mathrm{~W}]$ is nominal consumed active power; $V_{n}[\mathrm{~V}]$ is nominal voltage; $V[\mathrm{~V}]$ is actual RMS voltage value; $P$ $[\mathrm{W}]$ is actual consumed active power.

\section{Constant current load}

The consumed active power is changing directly to the voltage magnitude at the point in that model $P(V)$. Mathematical formula is introduced in eq. (2). If the voltage is increased by $\Delta V$, consumed active power is also increased according to this voltage change and vice versa. The consumed current remains constant during the voltage changes.

$$
\frac{P}{P_{n}}=\left(\frac{V}{V_{n}}\right)^{1}
$$
(1).

Where the meaning of quantities is the same as the eq.

\section{Constant power load}

In this case the magnitude and the character of consumed power do not depend on the voltage magnitude $P=$ const . This model has been mostly used in analysis [13]. Mathematical formula is described in eq. (3). If any voltage change occurs, the consumed active power or apparent power will not be changed.

$$
\frac{P}{P_{n}}=\left(\frac{V}{V_{n}}\right)^{0}=1,
$$
(1).

Where the meaning of quantities is the same as the eq.

\section{Polynomic load model}

Polynomic load model is nonlinear and is made up from a combination of all three nominal models. There are three identifying features- one which is not dependent on voltage, and two other, which depend on voltage linearly and squarely. Its mathematical formula is introduced in eq. (4). According to [13] that model is called as "ZIP" model. The sum of $a_{i}$ parameters has to be number one. It is very difficult to find all these parameters and it is possible to merely get somewhat close to their theoretically accurate values. 


$$
\frac{P}{P_{n}}=a_{1}+a_{2} \cdot\left(\frac{V}{V_{n}}\right)^{1}+a_{3} \cdot\left(\frac{V}{V_{n}}\right)^{2},
$$

Where:

$a_{1}, a_{2}, a_{3}[-]$ are constants and the meaning of other quantities is the same as the eq. (1).

\section{Simulation software DYNAST}

For the EV charging modelling the simulation software DYNAST was chosen. DYNAST is a software package for efficient and easy modelling, simulation and analysis of multidisciplinary nonlinear dynamic systems. It can be applied for numerical calculations in classical mechanics, heat transfer, energy transformation, electronics, electro-mechanics and other technical fields. Therefore, DYNAST can become a universal tool for various technical disciplines. DYNAST enables the user to define the models by a set of algebraic-differential equations, by block diagrams, by physical models using a multi-pole diagram or by a combination of all mentioned approaches. The multi-pole diagram also allows us to define actual physical structures and links among individual elements of the real system without deriving any equations or constructing any graphs [17-19].

\section{Measured and modified data from chosen charging station}

Measured data from various types of charging stations were used for the simulation purposes. The chosen type of charging station has the connector "Type 2 Mennekes", i.e. supply voltage $400 \mathrm{~V}, 3$ x $32 \mathrm{~A}$, and maximum output $22 \mathrm{~kW}$. The speed of charging with such power rating is considered to be fast. All data are measured at input into the charging station. It means a charging station with the EV battery behaves as a common electric device when connected to the electric power grid. Processes between the station and EV battery are not relevant for this method of modelling. All graph markers are point from average minute values. In the chosen case, voltage was slightly higher than nominal voltage $230 \mathrm{~V}$. Therefore, consumed currents are lower than supposed values $32 \mathrm{~A}$. The charging start is shown for time $5^{\text {th }}$ minute, and the end of charging happens after $134^{\text {th }}$ minute.

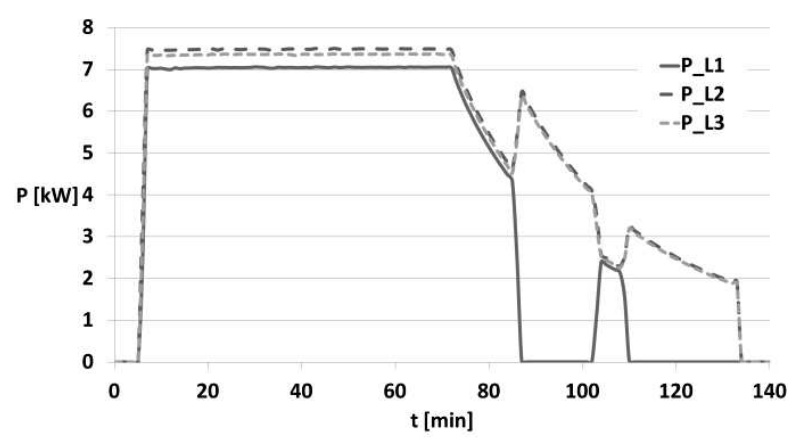

Fig. 1 Active power consumption of charging station

Consumed active powers by phases are shown in Fig. 1. A small asymmetry of power consumed is visible in individual phases, and it is caused by differential magnitudes of voltages, see this influence on constant active power between the $5^{\text {th }}$ and $72^{\text {nd }}$ minute, where the average active power is $7.29 \mathrm{~kW}$. The charging station communicates with the EV battery and searches for the best way to make charging the most effective. It can be seen between $85^{\text {th }}$ and $100^{\text {th }}$ minute, when phase one was switched off and the required power was consumed by the two other phases. The amount of consumed energy at nominal power level constitutes approximately $70 \%$ of all consumed energy while charging.

It is possible to obtain analogical processes for consumed currents. They have a similar time course, see Fig. 1. The average value of consumed current is $30.8 \mathrm{~A}$ between $5^{\text {th }}$ and $72^{\text {nd }}$ minute. During that time, consumed reactive power is close to $1 \mathrm{kvar}$ and decreases according to the active power decline. As the level of reactive power is significantly lower than active power, hence it was neglected for simplification. Simultaneously, that reactive power is consumed only by the charging station technology, but not by the battery in the vehicle.

RMS values of measured voltage are shown in Fig. 2. There are visible voltage drops which occur at load increase and also at a phase L1 disconnection. It can be seen that if the phase load is higher, the voltage on that phase is lower, for instance, at phase L2.

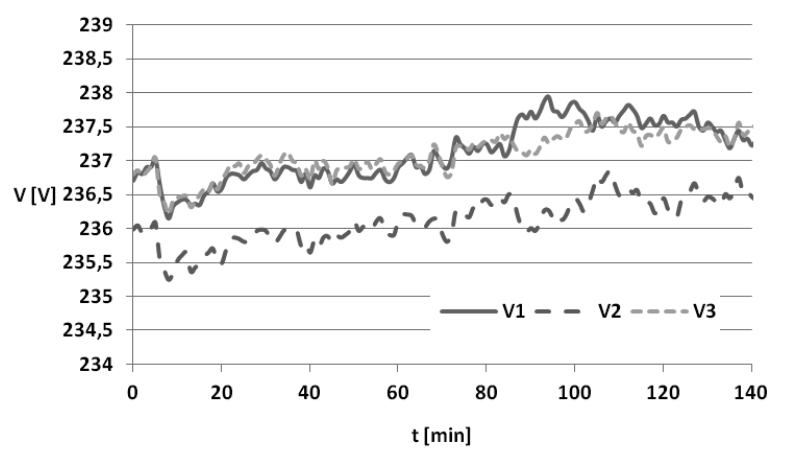

Fig. 2 RMS voltages when power consumption at charging station

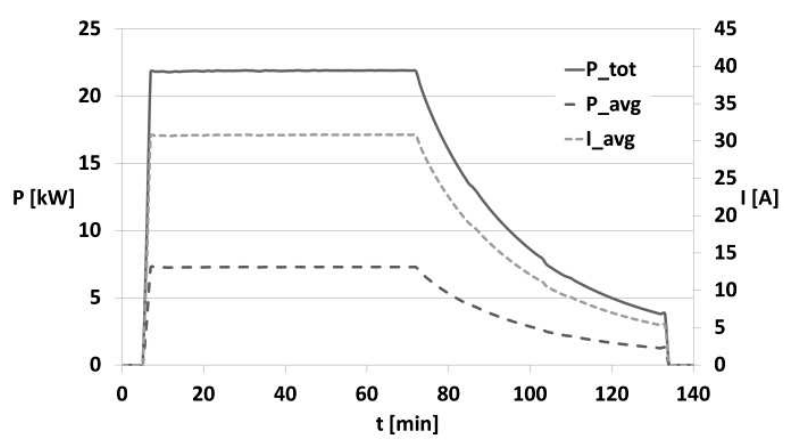

Fig. 3 Total active power consumption and average values of consumed active power and current

For the chosen way of modelling it is impossible to implement logic of switching phases into the basic properties as well as changes of consumed power, which are programmed into the control system of the charging unit. A simplification through usage of average values of current and active power is performed. The total and average 
consumed active power consumed by particular phases and average current are introduced in Fig. 3. Average current value is calculated from the real measured voltage and average power.

\section{Created load models}

Created models were applied in a simple one phase simulation circuit, see Fig. 4. The average values of power and current were used. The circuit consists of source of demanded voltage, and line impedance, which is simplified only to resistance because of voltage level and also two loads. The first load is an EC charging station, and the second one is a general charging station, both of them are resistive. A line impedance magnitude is either zero or $2 \mathrm{Ohms}$. Zero impedance means the the nominal voltage is directly connected to the load, while the non-zero impedance softens the voltage of the source. The general load is either $100 \mathrm{M} \Omega$ (it is not possible to set a zero value in the simulation program as the power consumed would be infinite) or $10 \Omega$. It leads to zero and approximately $2.3 \mathrm{~kW}$ power consumption of the general load respectively with nominal voltage.

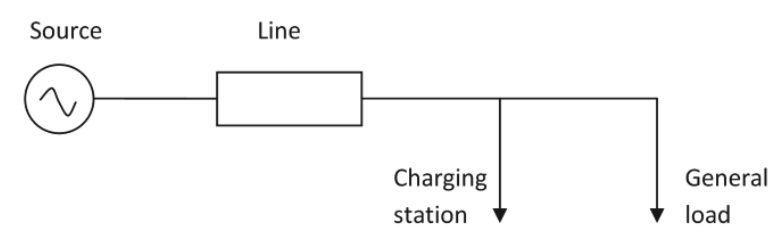

Fig. 4 Circuit diagram for testing of load models

\section{Resistive load model}

The progress of the values of load resistance depending on time is in fig. 5. The time scale is suitably chosen so that it enables one to run the simulation in convenient time and correspond to waveforms in Fig. 3. One minute of real charging time corresponds to the $0.01 \mathrm{~s}$ of simulation calculation. It does not really correspond to real-time charging of the EV, but in order to reach a quick calculation in the simulation software, it is necessary to decrease the scale. This scale was chosen with regard to proper function of indicators of electric values used in the simulation software.

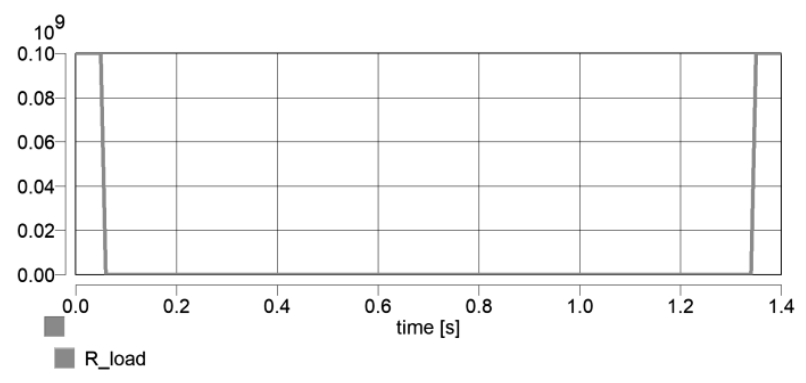

Fig. 5 Time behaviour of resistance change magnitude

If the line resistance and general load consumed power are zeros, time behaviour of voltage and current fully correspond to the measured data. If the line current loading is changed, voltage drop occur on it. Voltage on the load decreases, which leads to decline of consumed power or current. As an example the simulation results for this case are introduced in Fig. 6.

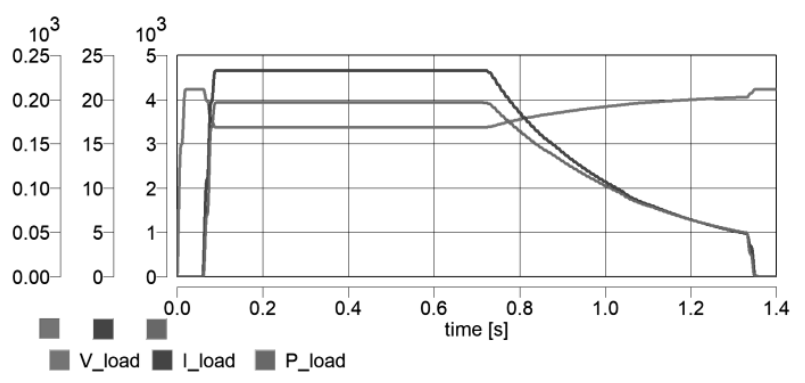

Fig. 6 Consumed current and active power by charging station

As soon as the load is connected, consumed current increases but does not reach the nominal value. Constant current magnitude is approximately $24 \mathrm{~A}$. It is caused by a voltage drop on the line. Without the general load, the voltage at the connection point is lower, approx $215 \mathrm{~V}$, see beginning of charging. With the load resistance decline, the line loading also decreases and voltage at the connection point rises. In the progress there is corrugation in the waveforms of all quantities in both the rising and decreasing phases of their change. It is caused by the activity of indicators of these quantities, which are originally created and used in the software. Their functioning and activity check are described by authors in [12, 17 - 19]. It means corrugation is not the model's fault or an error of calculation methods. The restive model appears as inappropriate as the nominal voltage at the point of connection is impossible to always reach. Voltage value is changing with the total loading of grid, as visible for example in Fig. 2.

\section{Current load model}

The current load model has implemented a control circuit inside, which changes internal resistance in dependence on voltage in order for the consumed current to reach predefined values. Its detailed working and structural description is mentioned in $[12,17]$.

There also has to be defined a non-zero value of active power or current during the simulation calculation because of singularity. It ensures a negligible value of active power or current in relevant time. Concurrently, the immense value of the load impedance has to be defined in one period of time because of the load model functions where the actual RMS voltage value is one input parameter. The resistance value is set at $100 \mathrm{M} \Omega$.

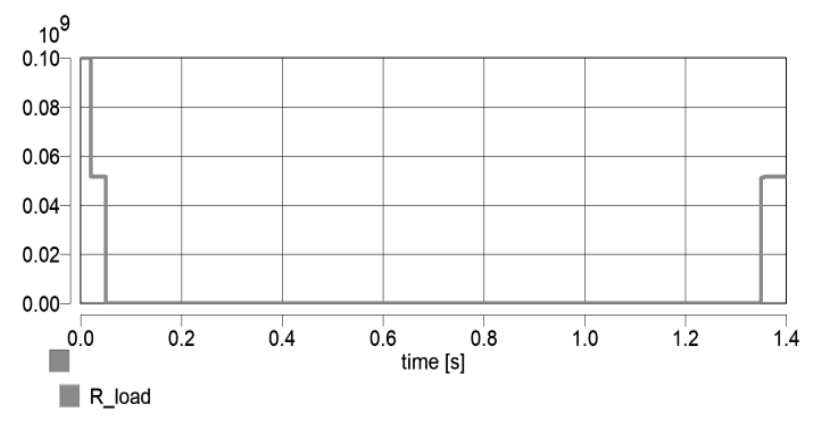

Fig. 7 Time dependence of resistance change magnitude 


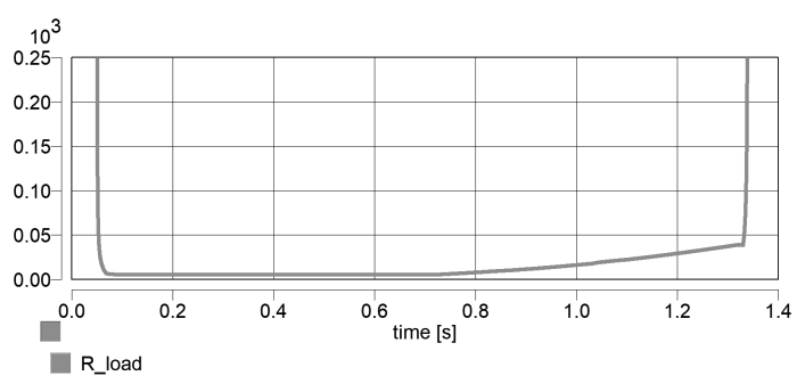

Fig. 8 The main part enlargement of time dependence of resistance change magnitude

For the zero value of general load and also line resistance results correspond to ones in Fig. 3. Actual resistance behaviour for the nonzero circuit parameters is shown in Fig. 7. Since a crucial part of the regulation of resistance value is impossible to read (low values), an enlargement of an important part of resistance behaviour is displayed in Fig. 8. It can be seen that while current decreases, the resistance increases in such a way in order to reach predefined current level.

Other waveform quantities for non-zero state are shown in Fig. 9. It is evident that consumed current behaviour matches preset tabular function. Although consumed active power has proper waveform, its value levels are lower. It is again caused by a voltage drop, which leads to influence on $\mathrm{V}_{\mathrm{RMS}}$ and therefore on total consumed active power. As the total consumed active power is inaccurate, this model is unsuitable. It is impossible to always reach nominal voltage at the desired point.

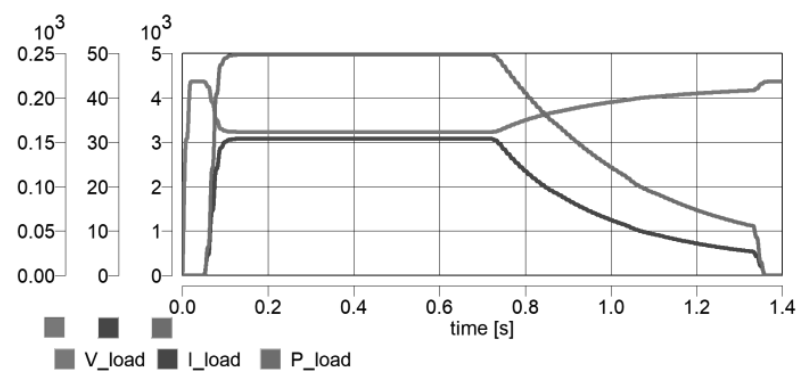

Fig. 9 Consumed current and active power by current load

\section{Power load model}

This model uses the same principle of resistance change when voltage changes as former model. Active power has to correspond to predefined values. Its detailed functioning and structural description are introduced in $[12,17]$.

For the zero value of general load and also line resistance, results correspond to ones in Fig. 3 again. When the supply circuit is not ideal, problems with self-regulation of voltage deviations occur. If the active power change is too steep, the circuit may over regulate, which leads to innacurate results. It is the case around $5^{\text {th }}$ minute of charging when within a minute or two there is a steep increase of nominal power. The result of this problem causes resistance to decrease to a very low value, which in return affects the current to rise steeply. Such current increment causes voltage growth drop on line etc. At the end voltage can drop to zero value and extreme current loading can occur. That sensitivity of the regulation circuit clearly limits usage of this model type. A possible elimination of this issue may be made by enlargement of the time scale (e.g. $14 \mathrm{~s}$ or more) for the simulation. However, such long-term simulation is currently unsuitable.

With the change of circuit charge, line resistance to $1.9 \Omega$ and disconnection of general load, the load model will operate. Simulation results are presented in Fig. 10. It can be seen that load model consumes desired active power all the time. Sharp power changes make a round round shape on the curve, for instance, around $7^{\text {th }}$ minute, and they are caused by the internal regulation circuit. As the voltage in the connection point is lower, the load consumes higher current. It means that the power load model is also unsuitable.

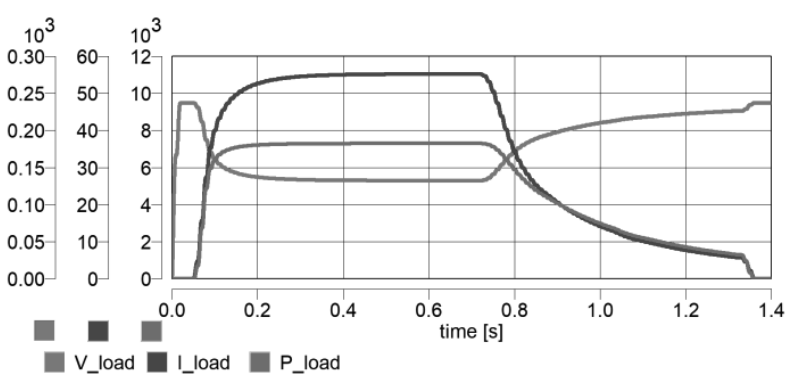

Fig. 10 Consumed current and active power by power load

\section{Polynomic load model}

This model has to have all the parameters defined precisely. If the influence in the current part predominates, the consumed power will be low. If the influence in the power part predominates, overregulation of the circuit may occur. A choice of appropriate levels of constants enables one to reach relatively sufficient results. For example, with zero line resistance and general load, the model behaviour is relatively good provided the parameters are $a_{1}=0.25, a_{2}=0.7, a_{3}=0.05$, see Fig. 11 . In case of small line resistance (approx $0.5 \Omega$ ), new parameter values are necessary. In this case, when $a_{1}=0.05, a_{2}=$ $0.9, a_{3}=0.05$, power consumption corresponds, but the consumed current is higher, which makes the polynomic model unsuitable as well.

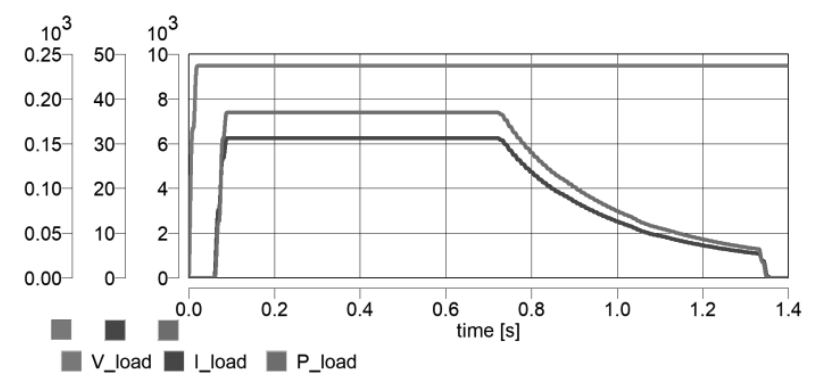

Fig. 11 Consumed current and active power by polynomic load

\section{Application of load models}

Although it has been found that any mentioned and applied model cannot be used as universal, it is possible 
to used them sufficiently and reliably while solving selected issues. When only one quantity is to be solved while regarding the other to be unimportant, some models can be applicable. In another case, while studying more physical quantities at the same time, parallel circuit and calculation would have to be created with the respective load model. It can be assumed restive and polynomic load models are inappropriate for desired simulations.

\section{Current load model}

For instance, this model can be sufficiently used to solve current loading of electrical distribution lines. The consumed current has directly defined values and therefore the current loading distribution within the lines is not influenced. Furthermore, the voltages in network points and their angles could be solved. In addition, active power lines losses could be sufficiently solved as long as their calculation is based on the solution of current magnitudes and electric line resistances.

\section{Power load model}

This model could be used for the power flow analysis in power grids. Simulation outputs would be affected by errors of individual powers because of incorrect current load. Moreover, power losses could be solved but the inaccuracy of results would be much higher. As a result, application of this model is very limited by voltage deviations. They would have to be very small to avoid over re-regulation of the load model regulation circuit. However, this model should be suitable and relatively accurate for measuring the level and character of consumed powers at grid points without relation to power flow in the grid.

\section{Conclusions}

Modelling of the EV charging as general load connected to the grid by using a physical diagram is possible. The current load model is relatively sufficient while the other tested models are mostly inaccurate or do not work in all cases. Mainly, the current load model is usable to solve current and voltage relations in electrical grids. It was found that no model can be used universally for the demanded simulations.

Problematics of the EV charging by physical diagram could be improved by implementing consumed reactive power of the charging station with the time defined dependency of this consumed power. The time behaviour of current or active power could be substituted by suitable curves with respective mathematical descriptions. The most precise model would need to have a system of control and the ability to reach the control circuit of the EV charger. Such a model could be used for more complex simulations to address selected matters.

\section{Acknowledgement}

This paper was funded and supported by an internal grant-UJEP-IGA-TC-2019-48-03-2: Modelling of specific elements, sources and loads in distribution power systems while respecting the quality and transmission of electrical energy.

\section{References}

[1] CHAN, M. S. V., CHAU, K. T., CHAN, C. C. (1998). Modeling of electric vehicle chargers. In: Proceedings of the $24^{\text {th }}$ Annual Conference of the IEEE Industrial Electronics Society, Vol. 1, pp. 433-438. Aachen, Germany.

[2] ZHANG T., CHEN, X., YU, Z., ZHU, X., SHI, D. (2018). A Monte Carlo Simulation Approach to Evaluate Service Capacities of EV Charging and Battery Swapping Stations. In: IEEE Transactions on Industrial Informatics, Vol. 14, No. 9, pp. 3914-3923.

[3] SHENAI, K., SHAH, K. (2011). Smart DC microgrid for efficient utilization of distributed renewable energy. In: Proceedings of 2011 IEEE EnergyTech, pp. 1-6. Cleveland, USA.

[4] COLLIN, J., DJOKIC, S. Z., THOMAS, H. F., MEYER, J. (2011). Modelling of electric vehicle chargers for power system analysis. In: Proceedings of $11^{\text {th }}$ International Conference on Electrical Power Quality and Utilisation, pp. 1-6. Lisbon, Portugal.

[5] KONGJEEN, Y., BHUMKITTIPICH, K. (2016). Modeling of electric vehicle loads for power flow analysis based on PSAT. In: Proceedings of $13^{\text {th }}$ International Conference on Electrical Engineering/Electronics, Computer, Telecommunications and Information Technology, pp. 1-6. Chiang Mai, Thailand.

[6] IGWE, E. W. (2016). Cable Sizing and Its Effect on Thermal and Ampacity Values in Underground Power Distribution. Dissertation. University of Kentucky, p. 58.

[7] ŠNAJDR, J., SEDLÁČEK, J., VOSTRACKÝ, Z. (2014). Application of a line ampacity model and its use in transmission lines opertions. Journal of ELECTRICAL ENGINEERING, Vol. 65, No. 4, pp. 221-227. ISSN 1335-3632

[8] SAMBA, A. (2015). Battery Electrical VehiclesAnalysis of Thermal Modelling and Thermal Management. Dissertation. Université de caen Basse Normandie, p. 203.

[9] OČENÁŠEK, V., LUŠTINEC, J., CIESLAR, M., BAJCURA, M. (2018). Application of Electrical Conductivity Measurement in Materials Research and in Solution of Technological Problems. In: Manufacturing Technology, Vol. 18, No. 5, pp. 805-810. ISSN 1213-2489

[10] SVOBODA, M., SOUKUP, J. (2013). Verification of Numeric Solution by Experiment for Examination Vertical Oscillation of a Mechanical System. In: Manufacturing Technology, Vol. 13, No. 4, pp. 559-563. ISSN 1213-2489

[11] KLIMENDA, F., RYCHLÍKOVÁ, L., PETRENKO, A., SVOBODA, M. (2015). Investigation of Vertical Vibration of a Vehicle Model 
Driving Through a Horizontal Curve. In: Manufacturing Technology, Vol. 15, No. 2, pp. 143-148. ISSN 1213-2489

[12] SÍŤǍr, V. (2015). Tvorba modelovacich analytických nástrojů v oboru elektroenergetiky s dirazem na oblast a spolehlivosti a provozu venkovních vedení. Dissertation. University of West Bohemia in Plisen, p. 137.

[13] MOTA, L. T. M., MOTA, A. A. (2007). New Trends on Load Modelling and Forecasting: Models and Techniques. Electric Power: Generation, Transmission and Efficiency. $1^{\text {st }}$ ed. Chapter 5, pp. 201-222. Nova Science Publisher, New York.

[14] KERSTING, W. H. (2007). Distribution System Modeling and Analysis. $2^{\text {nd }}$ ed. p. 421. CRC Press, Boca Ranton.

[15] LINDÉN, K., SEGERQUIST, I. (1993). Modelling of Load Device and Studying Load/System Characteristics, Technical Report No. 131L - revised edition. p. 138. Göteborg.
[16] NAVARRO, I. R. (2002). Dynamic Load Models for Power Systems: Estimation of Time-Varying Parameters during Normal Operation. Licentiate Thesis. Lund University, p. 158.

[17] SÍŤǍr, V., NOHÁČ, K., VELEBA, J. (2015). Statické modely zátěže a jejich modelování v netradičním softwaru pro elektroenergetiku. In: ElectroScope, Vol. 1, University of West Bohemia in Pilsen, p. 7. ISSN 1802-4564

[18] NOHÁČ, K., SÍŤǍ̆, V., VELEBA, J. (2016). Load Flow Analysis in Alternative Tools Using Equations, Physical and Block Diagrams. In: Proceedings of the $17^{\text {th }}$ international Scientific Conference - Electric Power Engineering 2016, pp. 1116. Prague, Czech Republic.

[19] SÍŤǍ̌, V., VELEBA, J., NOHÁČ, K. (2017). Load Slow Analysis Using Transformers Models in Alternative Simulation Tools. In: Proceedings of the $18^{\text {th }}$ international Scientific Conference Electric Power Engineering 2017, pp. 720-725. Ostrava, Czech Republic. 